\title{
Genome sequence of Vibrio sp. SALL 6 isolated from shellfish
}

Vengadesh Letchumanan $^{1 *}$, Hooi-Leng Ser ${ }^{1,2}$, Tan Wen-Si ${ }^{3,4}$, Kok-Gan Chan ${ }^{4,5}$, Nurul-Syakima Ab Mutalib $^{6}$

${ }^{1}$ Novel Bacteria and Drug Discovery Research Group (NBDD), Microbiome and Bioresource Research Strength, Jeffrey Cheah School of Medicine and Health Sciences, Monash University Malaysia, 47500 Bandar Sunway, Selangor Darul Ehsan, Malaysia

${ }^{2}$ Institute of Biomedical and Pharmaceutical Sciences, Guangdong University of Technology, Guangzhou, 510006, P. R. China

${ }^{3}$ Illumina Singapore Pte Ltd, Woodlands Industrial Park E1, Singapore

${ }^{4}$ Division of Genetics and Molecular Biology, Institute of Biological Sciences, Faculty of Science, University of Malaya, 50603 Kuala Lumpur, Malaysia

${ }^{5}$ International Genome Centre, Jiangsu University, Zhenjiang 212013, PR China

${ }^{6}$ UKM Medical Molecular Biology Institute (UMBI), UKM Medical Centre, University Kebangsaan Malaysia, Kuala Lumpur, Malaysia

\begin{abstract}
Members of the Vibrionaceae family are well known as foodborne pathogen that cause hazard to human in many forms of clinical infection and also affecting aquaculture via infection to livestock. This pathogen has caused seafood associated gastroenteritis cases in many countries including United States, Asian, and South East Asian countries. Antibiotics are usually used as prophylactic and therapeutic to manage the rising Vibrio infections, however, this in turn led to emergence of antibiotic resistant strains in the environments. Vibrio sp. SALL 6 isolated from shellfish was selected for genome sequencing to further explore its antimicrobial traits. Here, a high-quality genome sequence of Vibrio sp. SALL 6 is reported, while its genome reveals a potential for future antibiotic resistance managements.
\end{abstract}

Keywords: Vibrionaceae; foodborne; gastroenteritis; antibiotics; genome

Received: $26^{\text {th }}$ October 2019

Accepted: $26^{\text {th }}$ November 2019

Published Online: 06 ${ }^{\text {th }}$ December 2019
*Correspondence: Vengadesh Letchumanan, Novel Bacteria and Drug Discovery Research Group (NBDD), Microbiome and Bioresource Research Strength, Jeffrey Cheah School of Medicine and Health Sciences, Monash University Malaysia, 47500 Bandar Sunway, Selangor Darul Ehsan, Malaysia; lvengadesh@yahoo.com.

Citation: Letchumanan V, Ser H-L, Tan W-S, et al. Genome sequence of Vibrio sp. SALL 6 isolated from shellfish. Prog Microbes Mol Biol, 2019; 2(1): a0000044

\section{Introduction}

Vibrio species are natural inhabitant of aquatic environments and are the main cause of seafood-borne gastroenteritis ${ }^{[1,2]}$. This Gram-negative halophilic bacteria belong to the Vibrionaceae family ${ }^{[3-5]}$ and many of them are linked with aquatic animals such as crustaceans, molluscs and fish $^{[6]}$. Of the 12 identified pathogenic Vibrio sp., the three commonly reported are Vibrio cholerae and Vibrio parahaemolyticus - associated with seafood contamination, and Vibrio vulnificus - related via wound infections ${ }^{[7,8]}$. The increase in seafood consumption worldwide lead to the global rise of seafood production from aquaculture. This causes the marine animals to be prone to bacterial infections ${ }^{[9]}$. The occurrence of Vibrio sp. in our environments does raise a public concern on food safety due to the rising number of reported foodborne cases worldwide ${ }^{[10]}$. This situation has worsen by the emergence of antibiotic resistant bacteria which cause a delay in treatment, pro- long hospitalization and even mortality. Antibiotics are used in the aquaculture sector to treat bacterial infection, however, the misuse of them has resulted in the rising number of resistant foodborne pathogens such as Vibrio sp. ${ }^{[1-18]}$, Listeria sp. ${ }^{[19,20]}$, and Salmonella sp. ${ }^{[21-24]}$. Antibiotic resistance among foodborne pathogens is a major health issue and a great challenge to worldwide drug discovery programmes ${ }^{[25]}$. The clinical and environmental Vibrio sp. strains are reported to exhibit antibiotic resistance traits ${ }^{[26]}$. Hence, it is vital to continuously monitor and manage the occurrence of Vibrio in seafood and environments.

Vibrio sp. SALL 6 strain was isolated from shellfish originated from a wetmarket in Selangor, Malaysia. It formed green colony on thiosulphate citrate bile salt sucrose (TCBS) agar. The strain exhibited multidrug resistance profile towards 3/14 antibiotics tested. Based on the antibiotic susceptibility phenotype, Vibrio sp. SALL 
6 strain was resistant to ampicillin, $3^{\text {rd }}$ generation cephalosporin (cefotaxime) and aminoglycoside (amikacin). This is a distressing condition as the antibiotic resistant profile shown by this strain is among the recommended antimicrobials agents used in treatment of Vibrio sp. infections ${ }^{[27,28]}$. As an attempt to further explore its antimicrobial resistance traits, this strain was selected for genome sequencing.

\section{Data description}

Genomic DNA of Vibrio sp. SALL 6 was extracted using Masterpure ${ }^{\mathrm{TM}}$ DNA purification kit (Epicentre, Il lumina Inc., Madison, WI, USA) before performing RNase (Qiagen, USA) treatment ${ }^{[29,30]}$. The DNA quality was quantified using NanoDrop spectrophotometer (Thermo Scientific, Waltham, MA, USA) and a Qubit version 2.0 fluorometer (Life Technologies, Carlsbad, CA, USA). Illumina sequencing library of genomic DNA was prepared using Nextera ${ }^{\mathrm{TM}}$ DNA Sample Preparation kit (Illumina, San Diego, CA, USA) and library quality was validated by a Bioanalyzer 2100 high sensitivity DNA kit (Agilent Technologies, Palo Alto, CA) prior to sequencing. The genome of SALL 6 strain was sequenced on MiSeq platform with MiSeq Reagent Kit 2 $(2 \times 250 b p \text {; Illumina Inc, San Diego, CA, USA })^{[31]}$. The trimmed sequences were de novo assembled with CLC Genomic Workbench version 5.1 (CLC Bio, Denmark). Contigs with at least $200 \mathrm{bp}$ and 30 -fold coverage were selected for gene prediction and annotation. The bacteria identity was also checked by local BLAST against NCBI prokaryotic 16S rRNA database. Bacteria gene coding sequence (CDS) was predicted from the draft genome using Prodigal (version 2.6.1) ${ }^{[32]}$. Gene annotation was performed by local BLAST of translated predicted CDS against NCBI-nr database and also on Rapid Annotation using Subsystem Technology (RAST) server ${ }^{[33]}$. Presence of rRNA and tRNA genes were detected using RNAmmer and tRNAscan SE version $1.21^{[34,35]}$ A total of 81 contigs were generated with N50 size of 193,737 bp.
The assembled genome size of Vibrio sp. SALL 6 contains $4,989,632 \mathrm{bp}$, with an average genome coverage of 54fold with a $\mathrm{G}+\mathrm{C}$ content of $45.4 \%$ (Table 1 ). The whole genome project was deposited at DDBJ/EMBL/GenBank under accession MQVK00000000. The version described in this paper is the first version MQVK01000000. It is composed of 81 contigs and there were 4,500 protein coding genes (out of a total of 4,681 predicted gene) (Table $1)$.

Table 1. General features of Vibrio sp. SALL 6 draft genome

\begin{tabular}{ll}
\hline Attribute & Value \\
\hline Genome size (bp) & $4,989,632$ \\
G + C content \% & 45.4 \\
DNA scaffold & 81 \\
Total genes & 4,681 \\
Protein coding genes & 4,500 \\
RNA genes (5S, 16S, 24S) & $2,6,1$ \\
Pseudo genes & 66 \\
\hline
\end{tabular}

The analysis obtained from RAST server revealed 544 subsystems (Figure 1). The annotated genome has 74 genes responsible for resistance to antibiotic and toxic compounds including 28 genes for multidrug resistance efflux pumps, one gene for beta-lactamase, four genes for resistance to fluoroquinolones, and two genes for tetracycline resistance. The phenotypic resistance shown by Vibrio sp. SALL 6 toward ampicillin and cefotaxime is closely related to the gene coding beta-lactamase in the genome.

Multidrug resistance profile seen in the phenotype and genes of Vibrio sp. SALL 6 genome illustrates how extensive antibiotics have been utilized in agriculture and aquaculture settings. The efficacy of clinical antibiotics are declining, thus there is a need for non-antibiotic method such as bacteriophage application or natural plant antimicrobials to manage Vibrio infections in the aquaculture $^{[36,37]}$.

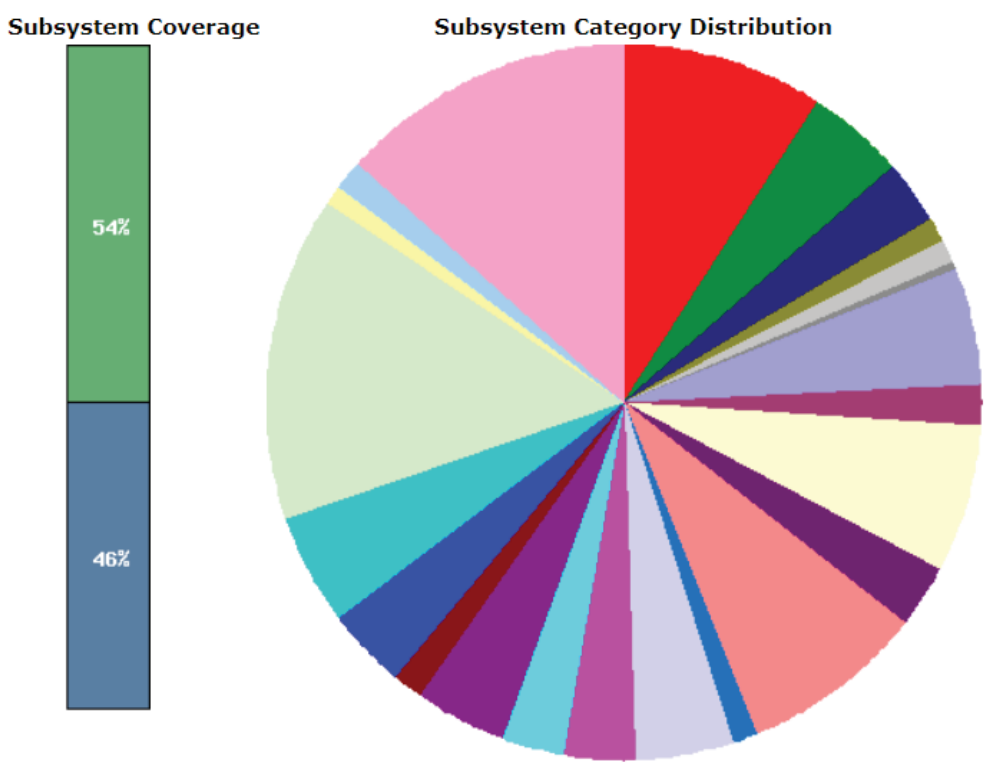

Subsystem Feature Counts

(⿴囗十) Cofactors, Vitamins, Prosthetic Groups, Pigments (336)

田 Cell Wall and Capsule (164)

$\boxplus$ Virulence, Disease and Defense (99)

$\boxplus$ Potassium metabolism (43)

$\boxplus$ Photosynthesis (0)

田 Miscellaneous (43)

由 Phages, Prophages, Transposable elements, Plasmids (11)

$\boxplus$ Membrane Transport (193)

$\boxplus$ Iron acquisition and metabolism (64)

( RNA Metabolism (245)

$\boxplus$ Nucleosides and Nucleotides (106)

$\boxplus$ Protein Metabolism (297)

$\boxplus$ Cell Division and Cell Cycle (41)

$\boxplus$ Motility and Chemotaxis (165)

$\boxplus$ Regulation and Cell signaling (119)

$\boxplus \square$ Secondary Metabolism (4)

$\boxplus$ DNA Metabolism (106)

Fatty Acids, Lipids, and Isoprenoids (147)

$\boxplus$ Nitrogen Metabolism (50)

田 Dormancy and Sporulation (3)

$\boxplus$ Respiration (138)

$\boxplus$ Stress Response (180)

田 Metabolism of Aromatic Compounds (8)

$\boxplus$ Amino Acids and Derivatives (533)

Sulfur Metabolism (31)

$\boxplus$ Phosphorus Metabolism (57)

$\boxplus$ Carbohydrates (462)

Figure 1. Subsystem category distribution of Vibrio sp. SALL 6 (based on RAST annotation server 


\section{Conflict of Interest}

The authors declare that the research was conducted in the absence of any commercial or financial relationships that could be construed as a potential conflict of interest.

\section{Acknowledgement}

This work was supported by University of Malaya for High Impact Research Grant (UM-MOHE HIR Nature Microbiome Grant No. H-50001-A000027 and No. A00000150001) and PPP Grant (PG090-2015B) awarded to K-GC.

\section{Reference}

1. Elmahdi S, DaSilva LV, and Parveen S. Antibiotic resistance of Vibrio parahaemolyticus and Vibrio vulnificus in various countries: A review. Food Microbiol, 2016; 57: 128-134.

2. Roux FL, Wegner KM, Baker-Austin C, et al. The emergence of Vibrio pathogens in Europe: Ecology, evolution, and pathogenesis (Paris, 11-12th March 2015). Front Microbiol, 2015; 6: 830.

3. Letchumanan V, Chan K-G, and Lee L-H. Vibrio parahaemolyticus: A review on the pathogenesis, prevalence, and advance molecular identification techniques. Front Microbiol 2014; 5: 705

4. Letchumanan V, Chan K-G, and Lee L-H. An insight of traditional plasmid curing in Vibrio species. Front Microbiol, 2015; 6: 735.

5. Sawabe T, Ogura Y, Matsumura Y, et al. Updating the Vibrio clades defined by multilocus sequence phylogeny: Proposal of eight new clades, and the description of Vibrio tritonius sp. nov. Front Microbiol, 2013; 4: 414 .

6. Bonnin-Jusserand M, Copin S, Le Bris C, et al., Vibrio species involved in seafood-borne outbreaks (Vibrio cholerae, V. parahaemolyticus and V. vulnificus): Review of microbiological versus recent molecular detection methods in seafood products. Crit Rev Food Sci Nutr, 2019; 59(4): 597-610.

7. Robert-Pillot A, Copin S, Himber C, et al. Occurrence of the three major Vibrio species pathogenic for human in seafood products consumed in France using real-time PCR. Int J Food Microbiol, 2014; 189: 75-81.

8. Raghunath P. Roles of thermostable direct hemolysin (TDH) and TDHrelated hemolysin (TRH) in Vibrio parahaemolyticus. Front Microbiol, 2015; 5: 805 .

9. Tan LT-H, Lee L-H, and Goh B-H, The bioprospecting of anti-Vibrio Streptomyces species: prevalence and applications. Prog Microbes Mol Biol, 2019; 1(1)

10. Ceccarelli D, Hasan NA, Huq A, et al. Distribution and dynamics of epidemic and pandemic Vibrio parahaemolyticus virulence factors. Front Cell Infect Microbiol, 2013; 3: 97.

11. Letchumanan V, Yin W-F, Lee L-H, et al. Prevalence and antimicrobial susceptibility of Vibrio parahaemolyticus isolated from retail shrimps in Malaysia. Front Microbiol, 2015; 6: 33

12. Letchumanan V, Pusparajah P, Tan LT-H, et al. Occurrence and antibiotic resistance of Vibrio parahaemolyticus from shellfish in Selangor, Malaysia. Front Microbiol, 2015; 6: 1417

13. Letchumanan V, Chan K-G, Khan TM, et al. Bile sensing: The activation of Vibrio parahaemolyticus virulence. Front Microbiol, 2017; 8: 728.

14. Lee L-H, Ab Mutalib N-S, Law JW-F, et al. Discovery on antibiotic resistance patterns of Vibrio parahaemolyticus in Selangor reveals carbapenemase producing Vibrio parahaemolyticus in marine and freshwater fish. Front Microbiol, 2018; 9: 2513.
15. Heng S-P, Letchumanan V, Deng C-Y, et al., Vibrio vulnificus: An environmental and clinical burden. Front Microbiol, 2017; 8: 997.

16. Law JWF, Letchumanan V, Chan KG, et al. Insights into detection and identification of foodborne pathogens. Food Borne Pathog Antibiot Resist, 2016.

17. Letchumanan V, Ab Mutalib N-S, Wong SH, et al. Determination of antibiotic resistance patterns of Vibrio parahaemolyticus from shrimp and shellfish in Selangor, Malaysia. Prog Microbes Mol Biol, 2019; 1(1).

18. Letchumanan V, Loo K-Y, Law JW-F, et al. Vibrio parahaemolyticus: The protagonist of foodborne diseases. Prog Microbes Mol Biol, 2019; 1(1).

19. Letchumanan V, Wong P-C, Goh B-H, et al. A review on the characteristics, taxanomy and prevalence of Listeria monocytogenes. Prog Microbes Mol Biol, 2018; 1(1).

20. Law JW-F, Ab Mutalib N-S, Chan K-G, et al. An insight into the isolation, enumeration, and molecular detection of Listeria monocytogenes in food. Front Microbiol, 2015; 6: 1227.

21. Eng S-K, Pusparajah P, Ab Mutalib N-S, et al. Salmonella: A review on pathogenesis, epidemiology and antibiotic resistance. Front Life Sci, 2015; 8(3): 284-293.

22. Khoo C-H, Cheah Y-K, Lee L-H, et al. Virulotyping of Salmonella enterica subsp. enterica isolated from indigenous vegetables and poultry meat in Malaysia using multiplex-PCR. Antonie Van Leeuwenhoek, 2009; 96(4): 441.

23. Yoke-Kqueen C, Learn-Han L, Noorzaleha A, et al. Characterization of multiple-antimicrobial-resistant Salmonella enterica subsp. enterica isolated from indigenous vegetables and poultry in Malaysia. Lett Appl Microbiol, 2008; 46(3): 318-324.24.

24. Learn-Han L, Yoke-Kqueen C, Salleh NA, et al. Analysis of Salmonella Agona and Salmonella Weltevreden in Malaysia by PCR fingerprinting and antibiotic resistance profiling. Antonie Van Leeuwenhoek, 2008; 94(3): 377.

25. Alanis AJ. Resistance to antibiotics: Are we in the post-antibiotic era? Arch Med Res, 2005; 36(6): 697-705.

26. Zavala-Norzagaray AA, Aguirre AA, Velazquez-Roman J, et al. Isolation, characterization, and antibiotic resistance of Vibrio spp. in sea turtles from Northwestern Mexico. Front Microbiol, 2015; 6 : 635.

27. Letchumanan V, Ser H-L, Chan K-G, et al. Genome sequence of Vibrio parahaemolyticus VP103 strain isolated from shrimp in Malaysia. Front Microbiol, 2016; 7: 1496

28. Letchumanan V, Ser H-L, Tan W-S, et al. Genome sequence of Vibrio parahaemolyticus VP152 strain isolated from Penaeus indicus in Malaysia. Front Microbiol, 2016; 7: 1410

29. Ser H-L, Tan W-S, Ab Mutalib N-S, et al. Draft genome sequence of mangrove-derived Streptomyces sp. MUSC 125 with antioxidan potential. Front Microbiol, 2016; 7: 1470.

30. Ser H-L, Tan W-S, Ab Mutalib N-S, et al. Genome sequence of Streptomyces mangrovisoli MUSC 149T isolated from intertidal sediments. Braz J Microbiol, 2018; 49(1): 13-15.

31. Kher H-L, Krishnan T, Letchumanan V, et al. Characterization of quorum sensing genes and $\mathrm{N}$-acyl homoserine lactones in Citrobacter amalonaticus strain YG6. Gene, 2019; 684: 58-69.

32. Hyatt D, Chen G-L, LoCascio PF, et al. Prodigal: Prokaryotic gene recognition and translation initiation site identification. $\mathrm{BMC}$ Bioinf, 2010; 11(1): 119.

33. Aziz RK, Bartels D, Best AA, et al. The RAST Server: Rapid annotations using subsystems technology. BMC Genomics, 2008; 9(1): 75 .

34. Lowe TM and Eddy SR. tRNAscan-SE: A program for improved detection of transfer RNA genes in genomic sequence. Nucleic Acids Res, 1997; 25(5): 955-964.

35. Lagesen K, Hallin P, Rødland EA, et al. RNAmmer: Consistent and rapid annotation of ribosomal RNA genes. Nucleic Acids Res, 2007; 35(9): 3100-3108

36. Letchumanan V, Chan K-G, Pusparajah P, et al. Insights into bacteriophage application in controlling Vibrio species. Front Microbiol, 2016; 7: 1114

37. Ma DS, Tan LT-H, Chan K-G, et al. Resveratrol - potential antibacterial agent against foodborne pathogens. Front Pharmacol, 2018; 9: 102. 